\title{
Why patients switch help from online to offline: An empirical investigation of emotions and effects of cognitions
}

\author{
Meng Yin \\ Xi'an Jiaotong University \\ yindameng@foxmail.com
}

\author{
Xiaoyu Xu \\ Xi’an Jiaotong University \\ xuxiaoyuxjtu@gmail.com
}

\begin{abstract}
People have different reactions when they encounter different levels of health threats. Individual cognitions towards the seriousness of health threat and emotions arise from health threats often motivate people to seek help online and offline. To examine the effects of cognitions and emotions on help seeking behaviors on different levels of health threats, we propose a research model based on protection motivation theory and cognition-emotion-behavior framework. Empirical data were collected through questionnaires and analyzed by Smart PLS. The results show that perceived susceptibility and perceived severity have positive significant influence on fear which leads on anxiety. Perceived health knowledge has negative influence on anxiety. Fear and anxiety can motivate individuals to seek help online and offline, especially in the high health threats condition. In addition, fear has significant influence on help seeking offline while anxiety has significant influence on help seeking online. Expectation confirmation has different effects on help seeking offline in different levels of health threats. This study extends PMT through the lens of cognition-emotion-behavior framework, and it expands research scope to help seeking behaviors.
\end{abstract}

\section{Introduction}

Help seeking behaviors including help seeking online and help seeking offline are two main approaches to fill their health information gaps and satisfy support needs [1]. With the rapid development of internet, internet provides users the access to adequate health-related information and becomes a most common channel for people to seek help online [2]. However, help from online are mainly healthrelated information, and in fact rarely solve health problems. The quality of health information online is not very perfect, which are often inconsistent, misleading and not trustworthy [3]. It is difficult for general users to solve health threats only through help seeking online. Hence, people often firstly search information online in order to obtain some understandings regarding certain health issue, such as through search engines, social media [4] and professional health websites. And then, people tend to ask for help from professionals, peers and families to obtain more reliable health information and suggestions $[5,6]$. Therefore, research on effects of cognitions and emotions on help seeking behaviors is significant for exploring and analyzing help seeking behaviors, and brings benefits for development and innovation of health-related service providers.

Prior literatures have examined critical factors determining the behaviors of help seeking online and offline. Four research gaps are identified and summarized from prior literatures. (1), Witte [7] and Kahlor [8] demonstrated that health threats can be regarded as one kind of threat appeals. And Protection Motivation Theory (PMT) was proposed by Rogers [9] as a framework investigating the impact of fear appeals, and has been noted as one of the most powerful explanatory theories for predicting individual protection intention and behaviors, such as health protection behavior $[10,11]$. Help seeking behavior is a type of protection behaviors, and aroused by health threats which can be regarded as a kind a fear appeal. However, little research has investigated help seeking behaviors based on PMT from threat appeal perspective. (2), Cognition-emotion-behavior is a basic framework which has demonstrated cognition has impact on emotion and emotion impacts behavior significantly [12, 13], and has been used for testing significant effect of emotion between cognition and behavior by scholars [14, 15]. But little research has been conducted to investigate effects of emotions in PMT based on cognition-emotion-behavior framework. (3), Fear and anxiety are two main negative emotions aroused by health threats and have been proved that they have significant influence on health help seeking behavior respectively by many scholars [2, 16, 17], but the effect of fear on anxiety and effects of fear and anxiety on help seeking online and offline are not clear in human behavior researches. (4), Most users firstly seek help online and then ask for help offline [16, 17], but scholars get opposite conclusions about the effects of help seeking online on help seeking offline [6]. And it is very necessary to further study the relationship 
between help seeking online and offline, and whether there are some mediators which play mediating effects between help seeking online and offline should also be explored.

Given above gaps, there are three questions should be answered in the following research. (1) When individuals encounter health threats, what are impact factors of help seeking behaviors? (2) Cognitions and emotions are generated from the judgment processes of health threats, but how cognitions affect emotions and how emotions affect help seeking behaviors are still unclear questions. (3) Does help seeking online decrease the probability of help seeking offline, are there any factors which exerts mediating effects between help seeking online and offline? In order to answer these questions, we put forward a research model based on PMT and cognition-emotion-behavior framework. And there are two judgment processes of health threats, the first stage is evaluating susceptibility and severity of health threats and then generates feeling of fear. The second stage is judging coping ability and comparing with fear, then generates anxiety $[7,8]$. In order to reduce uncomfortable feeling of fear and anxiety and to solve health threats, individuals usually ask for help from online and offline. Hence, we propose fear and anxiety to be factors of help seeking behaviors. Based on the conflicting results of effects of help seeking online on help seeking offline, we propose expectation confirmation of help seeking online may exert meditating effect between help seeking offline and help seeking offline. Then, empirical analysis is conducted to test research model and hypothesis, data are collected through questionnaires and the research results verify most of research hypothesis.

The rest of this paper is structured as follows. In the second section, we analyze the research context and theoretical background, and sum up previous studies about help seeking behavior and PMT. In the third section, we propose a research model and describe how we build the research model based on cognitionemotion-behavior framework and PMT, and put forward research hypothesis. In the fourth section, we develop a questionnaire to collect data, and analyze the data to test hypothesis through conducting structural equation model based on research model. In the fifth section, we describe the key findings, contributions and limitations of this study.

\section{Theoretical Background}

\subsection{Help seeking behavior}

Help seeking is a cognitive skill involving a series of actions including realizing the problems, identifying the needs of help, forming questions of needs and then choosing channels to solicit help [18, 19]. Help seeking can be identified as an effective getting strategy for acquiring information, getting support and solving problems with others' help [19]. People can get help from their family, friends, teachers, classmates and some other professionals to solve problems about life, learning, health, and etc [19, 20]. Developing of internet-related technologies has been influencing the way of help seeking, help seeking online becomes a main channel to solicit help, especially in the condition of rapid development of mobile internet and smartphone [21]. In summary, help seeking online and help seeking offline are two main help seeking approaches when people need help [20].

Factors of help seeking behaviors and the relationship between help seeking online and offline have been examined by many scholars. According to prior studies, cognitions and emotions are two main kinds of components determining help seeking behavior [22], individual cognitions are about health threats and abilities of threats coping, and emotions include positive and negative emotions such as hope, interest, fear, anxiety and worry [23]. And perceived information insufficiency, response efficacy, perceived vulnerability and self-efficacy are all important cognitions about health threats. Griffin [24] proposed a Model of Risk Information Seeking and Processing (RISP) to investigate preventive behavior, and defined three phases involving perceived individual characteristics, perceived information sufficiency and processing behavior. And cognitions including Perceived individual characteristics, perceived of current information and perceived information sufficiency had significant influence on processing behavior. Perceived information insufficiency is a very important cognition in help seeking behavior researches [8], but besides perceived information insufficiency, past experiences and attitudes, behavior control and beliefs are all cognitions which impact help seeking behaviors [25]. Response efficacy, selfefficacy, perceived vulnerability and perceived helpfulness have been demonstrated that they all are significant factors for health information seeking and healthy behavior [26]. And perceived relevance, searching self-efficacy and searching outcome expectancies are all factors of individual attitudes and behaviors which is demonstrated by Myrick [23].

Emotions are also significant components to explain help seeking behavior in the context of health threats, and consist of positive emotions and negative emotions [23]. The significant effects of emotions on help seeking behaviors have been proved by many scholars, fear and anxiety are considered as main emotion factors of help seeking behaviors. Griffin [24] 
demonstrated that affective response had important effect between perceived hazard characteristics and perceived need of information in RISP model [27], and the same effect is proved by Kahlor [8] in information seeking study. Myrick [23] proved that interested, fearful and hopeful were all antecedent variables of social cognitive, and then impacted attitudes and behaviors toward seeking. Anxiety is a consequence of uncertainty [28], and decrease the outcomes and efficacy [29]. Fear is important in health information seeking processes, and it has negative impact on searching outcomes, but positive influence on individuals' behavior intention [23], this effect is also proved in the security behavior research, fear positive impacts individuals' backup intention in the information security context of high fear-appeal [30]. Health anxiety is a significant factor of health information seeking and medical care utilization [31]. Individuals with high level of health anxiety have been shown to seek health information online at a greater frequency and for a longer duration than those with low levels of health anxiety $[32,33]$

\subsection{Fear and Anxiety}

Fear and anxiety are two main negative emotions in health behavior processes [23, 30], and they both have significant positive influences on help seeking behaviors. Fear can be defined as a "relational construct, aroused in response to a situation which is judged as dangerous and toward which protective action is taken" [34]. Fear as an emotional response which results from the personal and social perceptions of threats which observed, perceived and selfdiagnosed by individuals [35], has been proved it can impair individuals' ability to concentrate and succeed academically, as well as harm trust, cohesion and social control, and negative impact people's behavior such as coping behaviors and purchasing behaviors [36]. In the contrary, the effect of fear on individual protection behavior is positive, help seeking online [23] and protection behaviors are taken to reduce feeling of fear, solve threats and avoid potential harms [30]. Boss demonstrated that fear was an important consequence of perceived threats, and had a significant positive influence on individuals' protect behavior [30]. Pereira (2016) researched adolescents' help-seeking behavior, the results revealed fear positively associated with increased help-seeking [37], and Myrick (2017) got similar results from researches of health information seeking online [23].

Anxiety can be seen as being most often generated by concurrent and equivalent activation of fear [38], and it is another negative emotion which impact individuals' behaviors, Afifi (2004) had shown the influence of anxiety on outcomes, efficacy and behavior [29]. Psychological anxiety interferes with an individual's ability to accurately discriminate among stimuli, and individual's ability may be restricted by their prior expectations, experiences and knowledge, if individual has not adequate ability to cope with stimuli such as health threats, they will feel they can't control the situation which may harm them in the future [39]. Due to different stimuli, there are different kinds of anxiety, such as death anxiety [40], social anxiety [41], money anxiety [42] and health anxiety, and they all have influence on individuals' attitudes and behaviors. Health anxiety is a common and significant problem in those attending medical clinics and deserves greater awareness [43], and is the condition in which the individual believes that she or he has a serious disease, and this perceived serious illness has caused negative consequences, event thought she or he has no actual somatic illness [44]. Due to human's natural instinct of seeking benefit and avoiding damage, health anxiety has opposite effects on attitudes and behaviors compared with other kinds of anxiety [40, 41, 42 ]. When individuals have high level of health anxiety, they will have positive attitudes for help seeking behavior, and this effect is also proved by Lagoe [2] and Eastin [6].

Anxiety and fear are obviously overlapping, uncomfortable, negative emotion states generate from stimuli and both involve worry, apprehensive and negative feeling [45]. Since the different characteristics of fear and anxiety, they may generate different effects on help seeking behaviors. According to previous researches about fear and anxiety, four differences are summarized in many aspects such as definition, stimuli, function and tendency of behavior. (1) Fear refers to an emotional reaction which is brought about when a person experiences a situation which is threated. Anxiety involves an ambiguous feeling of fear and will cause uncertainly negative consequences [46]. (2) Fear is the response of observed stimuli which can be known by us such as data loss, but anxiety comes from uncertainly or unknown threats, and sometimes can't be controlled easily [28]. (3) Fear denotes dread of existing threats and it is "poststimuli" such as cancer and fatness. Anxiety is often "prestimuli" and it describes as an ineffable and unpleasant feeling of foreboding [45, 47], such as cancer may generate different terrible harms to health, but we don't know what harms they are and when they come out, and how terrible they are, especially we don't know how to treat them. (4) Fear has the function of moving animal away from danger. Individuals avoid damage of threats and get out of this situation to defend themselves. Anxiety has the function of moving animal toward danger, and 
motivates individuals to face with the threats and to control, solve the problems [38].

\subsection{Protection Motivation Theory}

Protection motivation theory (PMT) was developed by Rogers (1983) [48] from the health-related belief model (HBM) [49] in the social psychology and health domains [50], and was originally based on expectancyvalue theories and cognitive processes theories [51]. PMT consists of two aspects which refer to threat appraisal and coping appraisal, and then generate protection motivations and behaviors [48]. Threat appraisal explains how we evaluate threats, and includes perceived susceptibility and perceived severity [52]. And coping appraisal includes selfefficacy, response efficacy and response cost [48, 52].

PMT is proposed for the research of threat appeal, and has been noted as one of the most powerful explanatory theories for predicting individuals' protection intention and behaviors, such as domains of health protection behavior $[10,11]$, information systems security behavior [52] and usage behavior of health-related systems and services [53, 54]. Summarizing prior literatures, PMT can explain three kinds of health-related behavior: cease of unhealthy behavior, formation of health behavior and adoption behavior of health-related technologies and services. Sturges (1996) demonstrated that, for the adolescents and young adults, if they believed they can cope with the danger of tobacco use, high level of perceived threat resulted in increased intentions to refrain from tobacco use [55]. Threat appraisal and coping appraisal both arise from threat appeal such as messages or advises. Antismoking message was used for adolescents' nonsmoking intentions, and proved vulnerability to health risks, severity of social disapproval risks and self-efficacy all had positive influence on nonsmoking intention [10]. Besides wiping off people's unhealthy behaviors and habits, PMT can also be used to research formation of health behaviors. Plotnikoff (2002) studied the change of exercise behavior for the prevention of heart disease in a high risk [56], besides this, aerobic physical activity and resistance training was taken for treatment and management of type 2 diabetes and researched based on PMT [57]. Preventive behaviors of cancer is impacted by self-efficacy and cost, and can be explained by PMT partly [58]. With the rapid development of internet and technology, many healthrelated technologies come out, telemedicine, electronic health, mobile health, online health website and community are common for people to adopt. Lv [53] researched users' acceptance of mobile health services when they faced with health threats, results shown that response efficacy and self-efficacy had significant positive influences on usage intention of mobile health services for young and mid-age people [59]. In sum, PMT is effective for explaining individuals' healthrelated behaviors, and can be applied in different health-related behavior researches.

\section{Research model}

A help seeking behavior research model based on PMT and cognition-emotion-behavior framework was proposed as shown in figure 1. There are two evaluation stages when faced with health threats [8]. In the first stage, individuals evaluate the susceptibility and severity of health threats, and then fear generates from perceived susceptibility and perceived severity. In the second stage, individuals evaluate their own current coping abilities which involve health knowledge, health skills and information of diseases, and then compare with fear. Anxiety generates from the condition which individuals have no ability to cope with health threats and reduce the feeling of fear but with strong expectation of threats coping. In the second stage, we propose self-response efficacy which adapted from response efficacy of PMT and perceived health knowledge which adapted from perceived current knowledge of health information acquisition model (HIAM) [60] to be main components of coping ability and have significant influence on anxiety.

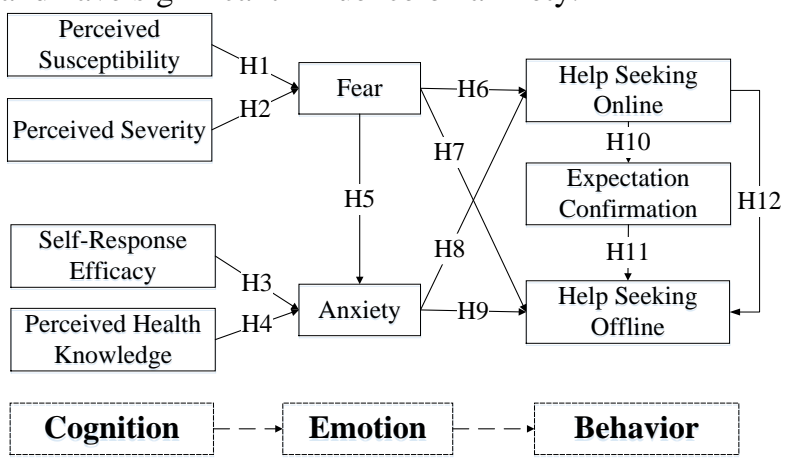

Figure 1 help seeking behavior research model

Fear and anxiety are proposed as significant influence factors of help seeking behaviors including help seeking online and help seeking offline in the research model. With the advantages of internet, help seeking online has been adopted by many users, but when individuals' needs can't be satisfied and expectations can't be confirmed, they will choose to ask for help offline. Help seeking offline especially visiting a doctor is regarded as a best way to solve health problems. Eastin (2006) investigated the relationship between health information seeking online and health care utilization behavior such as visiting a doctor, and found a negative relationship between information seeking online and help seeking offline [6]. But Norr [17] and Chae [16] got opposite results, 
which health information seeking online increased users' worry and anxiety, and led to ask for professional help offline. Based on expectation confirmation theory, we propose that the relationship is mediated by expectation confirmation, and when the expectation is confirmed, help seeking online will decrease help seeking offline. On the contrary, help seeking online will increases help seeking offline.

\subsection{Threat appraisal and coping appraisal to emotions}

Threat appraisal is the first process of health threats evaluation, involves perceived susceptibility and perceived severity [48]. Perceived susceptibility refers to the conditional probability that damage will occur without any behavior disposition in the context of health threats [61]. Perceived severity is evaluation of threats, refers to the degree of physical harm, psychological harm and dangers to others when faced with health threats. Individuals diagnose that they may get some kind of disease when they have analogous symptoms of some kind of disease, and then perceive the susceptibility and severity of the diseases. As Tanner (1991) demonstrated, fear is aroused by health threats, and has an important role in PMT, and can be impacted by threat appraisal [15]. Boss (2015) proved the significant influence of threat appraisal on fear when individual faced with high fear-appeal of data loss [30]. And fear arises from perceived susceptibility and perceived severity when faced with health threats. Therefore, we posit that,

H1: Perceived susceptibility of health threats has positive influence on fear.

$\mathrm{H} 2$ : Perceived severity of health threats has positive influence on fear.

Coping appraisal includes three factors in the PMT, response efficacy, response cost and self-efficacy [48]. But in the context of health threats, the second judgment process is regarded as the appraisal of their own ability of threats coping, and it is not the assessment of implementation ability and self-efficacy of protection behaviors. Therefore, response cost is dropped in this research model, and perceived selfresponse efficacy which is adapted from self-efficacy and response efficacy of PMT is proposed in this research model and refers to the belief about their own coping ability of health threats. Perceived health knowledge comes from HIAM [60], refers to perceived current health knowledge to deal adequately with health threats [8]. In prior researches, response efficacy and perceived health knowledge are both proved that they have significant influences on individual behavior. Anxiety generates from uncertain and unknown health threats, high self-response efficacy and perceived health knowledge will decrease feeling of anxiety. So, we posit hypothesis as below,

H3: Self-response efficacy has negative influence on anxiety.

H4: Perceived health knowledge has negative influence on anxiety.

Fear is defined as a negative emotion state, which generates from a person's experiences of the health threats, and expresses as worry, scare and fright. Anxiety is the ambiguous feeling of fear, and generates from uncertain and unknown health threats, refers to the emotion state of conflict situation which individuals have no enough ability to control health threats and reduce the feeling of fear but with strong expectation, and can't be sure the following consequences of the health threats [46]. So higher level of fear will bring about higher anxiety, and H5 is proposed as below,

H5: Fear has positive influence on anxiety.

\subsection{Emotions to behaviors}

Help seeking online refers to interaction with other people or internet health services in seeking health support and assistance to deal with health threats through internet, especially refers to health information seeking online [62]. Help seeking offline refers to get health information, treatment assistance from professionals in hospitals, professional health-related institutions. The goal of help seeking online is to acquire health-related information to reduce uncertain about health threats, then solve health threats. But the goal of help seeking offline is mainly for solving health threats and treatment of diseases, not just assessing to health-related information.

Fear and anxiety are both negative emotions generate from health threats, and people feel uncomfortable in the condition of fear and anxiety. Help seeking behaviors are effective approaches to reduce uncomfortable feelings. Boss [30], Pereira [37] and Myrick [23] all prove fear has significant positive influence on help seeking behaviors, that is to say high level of fear increase the probability of help seeking. So we propose fear prompt individuals to seek health help from online and offline. And we put forward hypothesis as below,

H6: Fear has positive influence on help seeking online.

H7: Fear has positive influence on help seeking offline.

Health-related anxiety is different from internet anxiety, social anxiety which have negative effect on individuals' attitudes and behaviors, as Eastin [6] and Lagoe [2] demonstrated, anxiety has positive influence on attitudes and help seeking behaviors. Anxiety is 
generated from fear and the uncertain health threats, and has the function of moving animal toward danger, and motivates individuals to face with the threats and to control, solve the problems [38]. Hence individuals search for health-related information online to reduce the uncertainty of health threats, and then face with them to solve health threats. So we posit that,

H8: Anxiety has positive influence on help seeking online.

H9: Anxiety has positive influence on help seeking offline.

\subsection{Help seeking online to offline}

The goal of help seeking is to fill health information gaps, reduce negative emotions, and solve the health threats. Usually, individuals seek help online first, and then ask for help offline. Eastin investigated the relationship between health information seeking online and health care utilization behavior such as visiting a doctor, and found a negative relationship between information seeking online and help seeking offline [6]. But Norr [17] and Chae [16] got opposite results, which health information seeking online increases users' worry and anxiety, and leads to seek help offline. Based on expectation confirmation theory, we believe the relationship is mediated by expectation confirmation, if the expectation is confirmed, help seeking online will decrease help seeking offline, on the contrary, help seeking online increases help seeking offline. So we posit that,

H10: Help seeking online has positive influence on expectation confirmation.

H11: Expectation confirmation has negative influence on help seeking offline.

H12: Help seeking online has positive influence on help seeking offline.

\section{Research methodology}

\subsection{Development of Questionnaires}

Data are collected through questionnaires, processed and analyzed by SPSS and Smart PLS. The questionnaire consist of three parts, the first part includes the description of questionnaire goals and contents and the definition of help seeking behaviors. The second part is the basic information survey of participants involve gender, age, education and personal disposable income. The third part are measures of latent variables. Due to our goal of this study is to test effects of cognitions and emotions on help seeking behaviors when faced health threats, we prompt participants to recall situations of health threats they faced in the latest year, such as disease symptoms they faced, and ask participants to answer questions based on their true experience of health threats. The level of health threats they faced is measured firstly, and then latent variables of research model are measured. Five-point Likert scale with anchors of strongly disagree one to strongly agree five for all items in our study was used. Measures of perceived susceptibility, perceived severity and self-response efficacy are adapted from Rogers [48] and Johnston [63]. Perceived knowledge is from Tormala [64], and measure of fear is adapted from Johnston [63]. Anxiety is mainly from Elhai [65] and Alkis [41]. And help seeking behaviors are adapted from Lagoe [2], and expectation confirmation is adapted from Bhattacherjee [66]. And all measures are all adapted based on the research context. As survey is conducted in China, measures of latent variables from previous researches are translated into Chinese firstly and then translated into English by two e-commerce scholars, and the questionnaire is confirmed after modifying many times.

\subsection{Data collection}

We collect pre-test data through wechat and then modify item descriptions of questionnaire based on the analysis of pre-test data, and then collect data through a questionnaire service website (www.sojump.com). 348 questionnaires are collected in total, and 43 questionnaires are deleted because of the time of filling out total questionnaire is below 100 seconds or contents of answer are conflict in the same item. At last, 305 valid questionnaires are received and the effective rate of questionnaires is $87.6 \%$. Male account for $41.64 \%$ and female account for $58.36 \%$. The main age group is between 19 and 45 years old, and account for $94.42 \%$. And degree of educations of $97.04 \%$ participants are above junior college. And Personal disposable income is mainly in the scope of 2001 and 10,000 Yuan, which account for $80 \%$.

\subsection{Measurement model}

In the process of data analysis, the mean, standard deviation (S.D.), factor loading and composite reliability (CR) are used for assessing the internal consistency of various measuring items, and Cronbach's $\alpha$ is used to measure the reliability of various measuring items. When the values of CR and Cronbach's $\alpha$ are all above 0.7 , it means measurement model have good internal consistency. We measure average variance extracted (AVE) to assess convergent validity, and regard various measuring items have good convergent validity when the value of AVE is above 0.5 . 
Confirmatory factor analysis is conducted to test the reliability and validity through the SPSS and Smart PLS programs and structural equation model (SEM) for path analysis according to the research model is also built through these software in this study. First of all, Cronbach's $\alpha$ of the whole data is measured through SPSS, and the value is 0.834 which is above 0.7 , so we think whole data has a high reliability. And then other scales are measured and shown in table 1. From table 1, all loadings are above 0.7 , and values of Cronbach's $\alpha$, values of CR and values of AVE all conform to the requirements of indicators, so all measuring factors have good reliability, internal consistency and convergent validity.

Table 1 descriptive statistics for the scales $(\mathrm{N}=305)$

\begin{tabular}{|c|c|c|c|c|c|c|}
\hline Item & $\begin{array}{l}\text { Mea } \\
n\end{array}$ & S.D. & $\begin{array}{l}\text { load } \\
\text { ing }\end{array}$ & $\begin{array}{l}\text { Cronbac } \\
\text { h's } \alpha\end{array}$ & $\mathrm{CR}$ & AVE \\
\hline PSU1 & 3.59 & 0.891 & 0.86 & \multirow{3}{*}{0.81} & \multirow{3}{*}{0.89} & \multirow{3}{*}{0.73} \\
\hline PSU2 & 3.40 & 0.992 & 0.85 & & & \\
\hline PSU3 & 3.00 & 1.048 & 0.85 & & & \\
\hline PSE1 & 3.40 & 1.060 & 0.93 & \multirow{3}{*}{0.92} & \multirow{3}{*}{0.95} & \multirow{3}{*}{0.86} \\
\hline PSE2 & 3.27 & 1.162 & 0.93 & & & \\
\hline PSE3 & 3.27 & 1.141 & 0.92 & & & \\
\hline HRE1 & 3.45 & 0.974 & 0.83 & \multirow{3}{*}{0.84} & \multirow{3}{*}{0.90} & \multirow{3}{*}{0.76} \\
\hline HRE2 & 3.24 & 1.016 & 0.90 & & & \\
\hline HRE3 & 3.58 & 0.938 & 0.89 & & & \\
\hline PK1 & 3.50 & 0.896 & 0.85 & \multirow{3}{*}{0.87} & \multirow{3}{*}{0.92} & \multirow{3}{*}{0.79} \\
\hline PK2 & 3.30 & 1.075 & 0.91 & & & \\
\hline PK3 & 3.23 & 1.005 & 0.91 & & & \\
\hline FER1 & 3.56 & 1.053 & 0.89 & \multirow{3}{*}{0.90} & \multirow{3}{*}{0.94} & \multirow{3}{*}{0.83} \\
\hline FER2 & 3.19 & 1.143 & 0.94 & & & \\
\hline FER3 & 2.93 & 1.197 & 0.91 & & & \\
\hline AXY1 & 3.32 & 1.072 & 0.92 & \multirow{3}{*}{0.92} & \multirow{3}{*}{0.95} & \multirow{3}{*}{0.86} \\
\hline AXY2 & 3.23 & 1.239 & 0.94 & & & \\
\hline AXY3 & 3.25 & 1.227 & 0.93 & & & \\
\hline HSON1 & 4.08 & 0.750 & 0.86 & \multirow{3}{*}{0.82} & \multirow{3}{*}{0.89} & \multirow{3}{*}{0.73} \\
\hline HSON2 & 4.06 & 0.761 & 0.84 & & & \\
\hline HSON3 & 4.11 & 0.762 & 0.87 & & & \\
\hline EC1 & 3.67 & 0.826 & 0.89 & \multirow{3}{*}{0.82} & \multirow{3}{*}{0.89} & \multirow{3}{*}{0.74} \\
\hline $\mathrm{EC} 2$ & 3.73 & 0.889 & 0.88 & & & \\
\hline EC3 & 3.67 & 0.953 & 0.81 & & & \\
\hline HSOF1 & 4.00 & 0.728 & 0.81 & \multirow{3}{*}{0.79} & \multirow{3}{*}{0.88} & \multirow{3}{*}{0.70} \\
\hline HSOF2 & 3.87 & 0.844 & 0.81 & & & \\
\hline HSOF3 & 3.95 & 0.794 & 0.90 & & & \\
\hline
\end{tabular}

PSU: Perceived Susceptibility; PSE: Perceived Severity; SRE: Self Response Efficacy; PHK: Perceived Health Knowledge; FER: Fear; AXY: Anxiety; HSON: Help Seeking Online; EC: Expectation Confirmation; HSOF: Help Seeking Offline.

In order to test the discriminant validity, we compared the square root of AVE and latent variable correlation coefficients. For each latent variable, the square root of AVE is significant larger than its correlation coefficients with other latent variables, which suggesting good discriminant validity.

As our data is collected from single respondents, common method bias could influence the validity of the results. In order to test such bias, we use Harman's single-factor test to check the CMB [67], and according to the results of factor analysis in the unrotated factor matrix, the variance explained by the first factor is $31.1 \%$. Therefore, CMB should not be a serious concern in this study.

\subsection{Structural model}

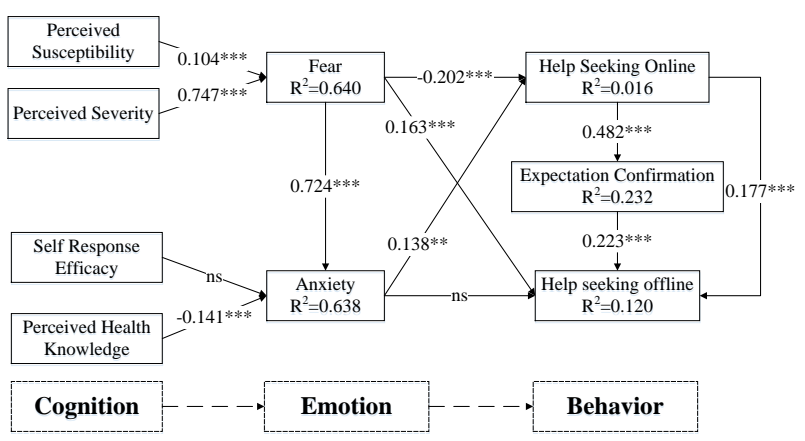

Figure 2 Results of research model: high health threat

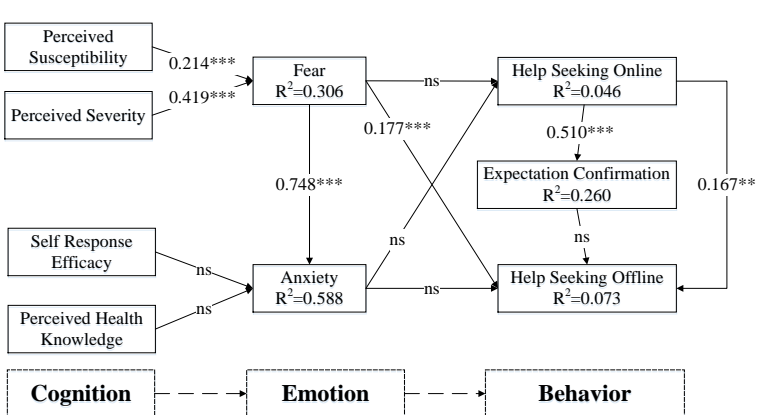

Figure 3 Results of research model: low health threat

According to the different level of health threats which reported by participants, data is divided into high health threat and low health threat parts. Smart PLS is used for building SEM, and PLS Algorithm is ran to test the path coefficients between latent variables, Bootstrapping is used for calculating the significance level. Figure 2 and figure 3 show the path coefficients and significance levels when faced with high health threat and low health threat.

\section{Discussion}

\subsection{Discussion of research results}


From the PLS results of research model and summary of hypothesis testing results, we can see individuals have different reactions when faced with different level of health threats. In the condition of high level of health threats, perceived susceptibility and perceived severity have significant positive influences on fear, $\mathrm{H} 1$ and $\mathrm{H} 2$ are supported. Fear has significant positive impact on anxiety which H5 are supported, and perceived health knowledge has negative influence on anxiety, but the effect of selfresponse efficacy on anxiety is not significant, H3 is not supported and $\mathrm{H} 4$ is supported. Fear has negative impact on help seeking online and has positive influence on help seeking offline which $\mathrm{H} 6$ is not supported and $\mathrm{H} 7$ is supported, that is to say individuals will choose to seek help from offline professionals without choosing to seek help from online when faced with high health threats. But anxiety has opposite results, which anxiety has positive significant influence on help seeking online which supports H8, and the effect of anxiety on help seeking offline is not significant which H9 is not supported, in other words, when individuals have feeling of anxiety when faced with high health threats, they will chose to know more about them without seeking help from offline with uncertain health threats. These difference effects of fear and anxiety can be explained by different characteristics of fear and anxiety, which fear has the function of moving animal away from danger and anxiety has the function of moving animal toward danger, and motivates individuals to face with the threats and to control, solve the problems [38]. Help seeking online has significant positive influence on help seeking offline, and has significant positive influence on expectation confirmation which then impact help seeking offline significantly, $\mathrm{H} 10$ and $\mathrm{H} 12$ are supported but $\mathrm{H} 11$ is not supported. That is, although individuals can get health help such38 as health information from online to fill their health information gaps, their health problems can't be solved based on online help since high level of health threats, individuals still seek help from offline professionals to avoid or solve health problems.

Comparing with high health threats, responses of individuals are not so much significant when faced with low health threats as shown in figure 3. Perceived susceptibility and perceived severity have significant positive influence on fear which then has significant influence on anxiety, but effects of self-response efficacy and perceived health knowledge on anxiety are not significant. In the condition of low health threats, emotions have low significant influence on help seeking behaviors, which only fear has significant positive influence on help seeking offline. Individuals may have enough health knowledge and self-response ability to cope with low health threats, and may have a little of anxiety feeling which has no significant impact on help seeking behaviors. When faced with low health threats, Individuals also seek help offline after seeking help online, but the significant level of help seeking online on help seeking offline is low. And help seeking online has significant positive influence on expectation confirmation which has no influence on help seeking offline, which can be understood as help seeking online can fill their needs of low health threats.

According to the above analysis, high health threats can arise fear and anxiety significantly which have significant influence on help seeking behaviors, but for low health threats, effects of cognitions of health threats on emotions are weak, and emotions also have weak influence on help seeking behaviors. Although help seeking online can confirm individuals' expectation, individuals still seek help from offline professionals when faced with high health threats. But in the condition of low health threats, individuals will stop asking for help once individuals' health information gaps and health support needs are satisfied. In general, whether high health threats or low health threats, the explanations of the variance in help seeking behaviors of fear and anxiety are very low, that is to say, although fear and anxiety have influences on help seeking behaviors, they are not main influence factors of help seeking behaviors.

\subsection{Theoretical contributions}

There are three theoretical contributions in this study. First, according to previous researches, we propose a help seeking behavior research model based on PMT and cognition-emotion-behavior, and verify the effects of cognitions and emotions on behaviors through empirical study. This study extends the protection motivation theory research, and emphasizes the important role of emotions in help seeking behavior researches. Cognition-emotion-behavior is a basic framework, which has been proved that it can be used in human behaviors research in the context of health threats, and we expand the research scope of cognitionemotion-behavior in this study.

Second, this study has demonstrates that fear and anxiety are aroused by health threats, and they play important roles in individual's behaviors. The results show that fear generates from threat appraisal in the first judgment stage and anxiety arises from perceived health knowledge and self-response efficacy in the second stage. These results give us a clear and deep understanding about fear and anxiety in health threats context. This study also demonstrates the relationship between fear and anxiety, and prove that fear has significant influence on anxiety. 
Third, the research model demonstrates expectation confirmation is a mediator variable between help seeking online and help seeking offline, and the results of empirical study show that help seeking online has significant influence on expectation confirmation, but the effects are difference in different levels of health threats. In the high level of health threats, even though expectations of help seeking online are confirmed, individuals still ask for help from offline professionals. But in the low level of health threats, if the expectations are confirmed through help seeking online, individuals will stop asking for help from offline. The level of health threats is a significant control variable which is proved in this study. In a word, this research fill prior research gaps of help seeking behavior through constructing a help seeking behavior research model based on PMT and cognition-emotion-behavior framework.

\subsection{Practical implications}

We can get three enlightenments for health education, health service providers and health-related institutions. First of all, perceived health knowledge is an important factor of anxiety, sufficient health-related knowledge can reduce the feeling of anxiety and increase confidence of coping when faced with health threats. Health education institutions should give much more health knowledge education to individuals to improve their level of health knowledge and their ability of health threats coping. Second, from this research, we can see threat appraisal, coping appraisal and negative emotions are all factors of help seeking behavior. Health service providers such as healthrelated information websites should analyze individuals' needs based on this research and develop reasonable business strategies and innovations of health services to satisfy individuals' needs and reduce negative emotion feelings. Third, expectation confirmation of help seeking online can't decrease the probability of help seeking offline. High quality of health information and treatment services needed to satisfy individuals' health needs and to decrease help seeking offline, which can decrease medical pressure and improve the medical efficiency of offline health institutions, and it will also improve the health level of whole society. Hence, Health-related institutions should provide professional online health services to individuals for solving their health threats, and try their best to satisfy individuals' a variety of health needs, so that help seeking online can improve individuals' expectation confirmation and decrease the probability of help seeking offline.

\subsection{Limitations and further researches}

There are some limitations in this study and some further researches should be done in the future. First, we collect data through survey, and the level of health threats are self-reported. This method interferes the true level of health threats they faced with, and then interferes the results of our research. We can use experiment method to control the level of health threats and collect data. Besides this, fear and anxiety are all reported by participants, and there may be some people who are not clear about the different feelings of fear and anxiety, and lead to inaccurate measurement results of fear and anxiety. Besides the limitation of data collect method, the sample size is also a limitation in our study, there are only 149 high health threats samples, and 156 low health threats samples. Hence, future research should collect much more data.

Second, men seek help less often than women and underutilize medical and mental health services [68], gender is an important control variable for help seeking behavior. Comparing with young people, elder people much more likely to visit doctors through traditional ways, age is another important control variable in this research. Visiting a doctor is very expensive for many low income people, but for rich people, time is much more important for them, and seeing a doctor is a best choose for them. Therefore, gender, age, education and income all can be regard as control variables.

Third, we abandon response cost which is from PMT in our research model, but it is important for individuals to judge and conduct protection behaviors. Response cost of help seeking online and help seeking offline are different, especially for different people. Response cost of help seeking online is high for elder people, and response cost of help seeking offline is high for low income people. In future research, the influence of response cost on help seeking behaviors should be tested.

Fourth, the relationship between help seeking online and help seeking offline is very complex. Although expectation confirmation exerts mediating effects between help seeking online and offline, but the hypothesis are not supported completely. And satisfaction of help seeking online may have significant influence on help seeking offline, and can be put into the model in the future research. And because of the low explanations of the variance in help seeking behaviors of fear and anxiety, there may be some other factors of help seeking online and help seeking offline, further research should be done in the future to explore other factors of help seeking behaviors.

\section{Acknowledgment}

This research was supported by China Ministry of Education under operation grant of 2016M602838. 


\section{References}

[1] C J. McKinley, P J. Wright. Informational social support and online health information seeking: Examining the association between factors contributing to healthy eating behavior. Computers in Human Behavior, 37, 2014, pp. 107116.

[2] C. Lagoe, D. Atkin. Health anxiety in the digital age: An exploration of psychological determinants of online health information seeking. Computers in Human Behavior, 52, 2015, pp. 484-491.

[3] S. Pant, A. Deshmukh, K. Murugiah, et al. Assessing the credibility of the "YouTube approach" to health information on acute myocardial infarction. Clinical cardiology, 35(5), 2012, pp. 281-285.

[4] H M. Asghar. Measuring Information Seeking through Facebook: Scale development and initial evidence of Information Seeking in Facebook Scale (ISFS). Computers in Human Behavior, 52, 2015, pp. 259-270.

[5] W Y. Lin, X. Zhang, H. Song, et al. Health information seeking in the Web 2.0 age: Trust in social media, uncertainty reduction, and self-disclosure. Computers in Human Behavior, 56, 2016, pp. 289-294.

[6] M S. Eastin, N M. Guinsler. Worried and wired: effects of health anxiety on information-seeking and health care utilization behaviors. CyberPsychology \& Behavior, 9(4), 2006, pp, 494-498.

[7] K. Witte. Theory-based interventions and evaluations of outreach efforts. Research review. National Network of Libraries of Medicine Pacific Northwest Region, Outreach Evaluation Resource Centre, Seattle, 1998.

[8] L A. Kahlor. PRISM: A planned risk information seeking model. Health Communication, 25(4), 2010, pp, 345356.

[9] R W. Rogers. A protection motivation theory of fear appeals and attitude change. The journal of psychology, 91(1), 1975, pp, 93-114.

[10] C. Pechman, G. Zhao, M E. Goldberg, et al. What to convey in antismoking advertisements for adolescents: The use of protection motivation theory to identify effective message themes. Journal of Marketing, 67(2), 2003, pp. 1-18.

[11] A. Gaston, H. Prapavessis. Using a combined protection motivation theory and health action process approach intervention to promote exercise during pregnancy. Journal of behavioral medicine, 37(2), 2014, pp. 173-184.

[12] B. Weiner. A theory of motivation for some classroom experiences. Journal of educational psychology, 71(1), 1979, pp. 3-25.

[13] B. Weiner, D. Russell, D. Lerman. The cognitionemotion process in achievement-related contexts. Journal of personality and social psychology, 37(7), 1979, pp. 12111220 .

[14] B. Weiner. A cognitive (attribution)-emotion-action model of motivated behavior: An analysis of judgments of help-giving. Journal of Personality and Social psychology, 39(2), 1980, pp. 186-200.

[15] JR J F. Tanner, J B. Hunt, D R. Eppright. The protection motivation model: A normative model of fear appeals. The Journal of Marketing,55(3), 1991, pp. 36-45.
[16] J. Chae. Online cancer information seeking increases cancer worry. Computers in Human Behavior, 52, 2015, pp. $144-150$.

[17] A M. Norr, D W. Capron, N B. Schmidt. Medical information seeking: impact on risk for anxiety psychopathology. Journal of behavior therapy and experimental psychiatry, 45(3), 2014, pp. 402-407.

[18] V. Aleve, B. Mclaren, I. Roll, et al. Toward metacognitive tutoring: A model of help seeking with a Cognitive Tutor. International Journal of Artificial Intelligence in Education, 16(2), 2006, pp. 101-128.

[19] Q. Hao, E. Wright, B. Barnes, et al. What are the most important predictors of computer science students' online help-seeking behaviors?. Computers in Human Behavior, 62, 2016, pp. 467-474.

[20] D. Reifegerste, M. Bachl, E. Baumann. Surrogate Health Information Seeking in Europe: Influence of Source Type and Social Network Variables. International Journal of Medical Informatics, 103, 2017, pp. 7-14.

[21] E H. Shortliffe. Health care and the next generation Internet[J]. Annals of Internal Medicine, 129(2), 1998, pp. 138-140.

[22] C C. Kuhlthau. Inside the search process: Information seeking from the user's perspective. Journal of the American Society for information Science, 42(5), 1991, pp. 361-371.

[23] J G. Myrick. The role of emotions and social cognitive variables in online health information seeking processes and effects. Computers in Human Behavior, 68, 2017, pp. 422-433.

[24] R J. Griffin, S. Dunwoody, K. Neuwirth. Proposed model of the relationship of risk information seeking and processing to the development of preventive behaviors. Environmental research, 80(2), 1999, pp. S230-S245.

[25] C. Marton, C. Wei Choo. A review of theoretical models of health information seeking on the web. Journal of Documentation, 68(3), 2012, pp. 330-352.

[26] C J. McKinley, P J. Wright. Informational social support and online health information seeking: Examining the association between factors contributing to healthy eating behavior. Computers in Human Behavior, 37, 2014, pp. 107116.

[27] Z J. Yang, L A. Kahlor. What, me worry? The role of affect in information seeking and avoidance. Science Communication, 35(2), 2013, pp. 189-212.

[28] C E. Izard. The psychology of emotions. Springer Science \& Business Media, 1991.

[29] W A. Afifi, J L. Weiner. Toward a theory of motivated information management. Communication Theory, 14(2), 2004, pp. 167-190.

[30] S R. Boss, D F. Galletta, P B. Lowry, et al. What do users have to fear? Using fear appeals to engender threats and fear that motivate protective security behaviors. MIS Quarterly, 39(4), 2015pp. 837-864.

[31] M S. Eastin, N M. Guinsler. Worried and wired: effects of health anxiety on information-seeking and health care utilization behaviors. Cyber Psychology \& Behavior, 9(4), 2006, pp. 494-498.

\section{Reference Available Upon Request}

